\title{
PERFORMANCE MEASUREMENTS OF JET PUMPS WITH MULTIPLE HOLES
}

\author{
J.P. Oosterhuis ${ }^{1 *}$, S. Bühler ${ }^{1}$, D. Wilcox ${ }^{2}$, and T.H. van der Meer ${ }^{1}$ \\ ${ }^{1}$ Thermal Engineering, Engineering Technology, University of Twente, P.O. Box 217, 7500AE, Enschede, The Netherlands. \\ *Corresponding author's e-mail: j.p.oosterhuis@utwente.nl \\ 2 Chart Inc., Troy, New York, USA.
}

Keywords: jet pumps, minor losses, flow separation, acoustic streaming

\section{Introduction}

In order to cancel Gedeon streaming in traveling wave thermoacoustic devices, a jet pump can be used $[2,3]$. Due to its asymmetric shape an asymmetry in the minor losses during each acoustic cycle occurs that yields a time-averaged pressure drop across the jet pump. By balancing the time-averaged pressure drop across the jet pump with that which exists across the regenerator of a thermoacoustic device, Gedeon streaming can be suppressed.

The current state of the art in jet pump design methodology is based on a quasi-steady approximation [2]. In a recent numerical study we have shown that the applicability of this approximation is limited [4]. Currently, the previous numerical work is extended to the experimental domain to investigate the influence of three-dimensional geometry variations. By "splitting" a jet pump with a single hole into a geometry with multiple parallel holes, the size of the jet pump can be reduced while maintaining the taper angle and the total cross-sectional area. Reducing the size of the jet pump will aid in the design of compact thermoacoustic engines.

\section{Method}

The experimental setup is shown schematically in Figure. 1 and is similar to the setup previously used by Aben [1]. A $40 \mathrm{~Hz}$ acoustic wave is generated using a loudspeaker. Both the pressure amplitude $p_{1}$ and the time-averaged pressure $p_{2}$ are measured at four different locations as indicated by P1-P4 in Fig. 1. From the measured pressure amplitude the velocity amplitude inside the jet pump $u_{1, J P}$ and the acoustic power dissipation $\Delta \dot{E}_{2}$ are calculated. Eight different jet pump samples are investigated having a various number of holes $(1,2,4,8$ and 16) and two different taper angles $\left(7^{\circ}\right.$ and $\left.15^{\circ}\right)$. The total cross-sectional area is kept constant at both openings and the jet pump length is varied in order to maintain the correct taper angle among the different samples.

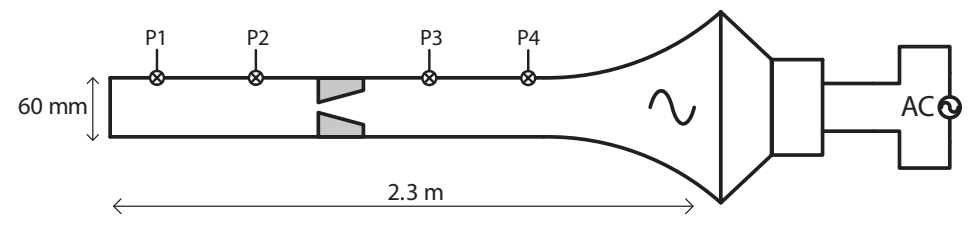

Figure 1: Schematic of experimental setup with pressure sensors and jet pump sample indicated. Dimensions not to scale.

\section{Results and discussion}

Figure. 2 shows the jet pump performance in terms of the dimensionless pressure drop and acoustic power dissipation which are defined using [5],

$$
\Delta p_{2}^{*}=\frac{8 \Delta p_{2}}{\rho_{0}\left|u_{1, J P}\right|^{2}}, \quad \Delta \dot{E}_{2}^{*}=\frac{3 \pi \Delta \dot{E}_{2}}{\rho_{0} A_{s}\left|u_{1, J P}\right|^{3}},
$$


with $A_{s}$ the total cross-sectional area at the jet pumps small opening. On the horizontal axis, $K C_{D}$ is the Keulegan-Carpenter number based on the diameter of the small jet pump opening and represents the wave amplitude. While the samples with one or two holes ( and $\mathbf{\square})$ show a comparable performance for $K C_{D}>10$, the pressure drop is significantly decreased for jet pump samples with 4 or more holes $(\nabla, \downarrow$ and $\star)$. This is observed for both the $7^{\circ}$ and $15^{\circ}$ taper angles. However, the time-averaged pressure drop for the $15^{\circ}$ taper angle is generally lower which is probably caused by stronger flow separation in the diverging flow direction. The dimensionless acoustic power dissipation shows a mixed trend. Albeit the 16 hole jet pump shows higher acoustic power dissipation compared to the single or double hole jet pump samples, a definitive trend could not be distinguished based on these results.
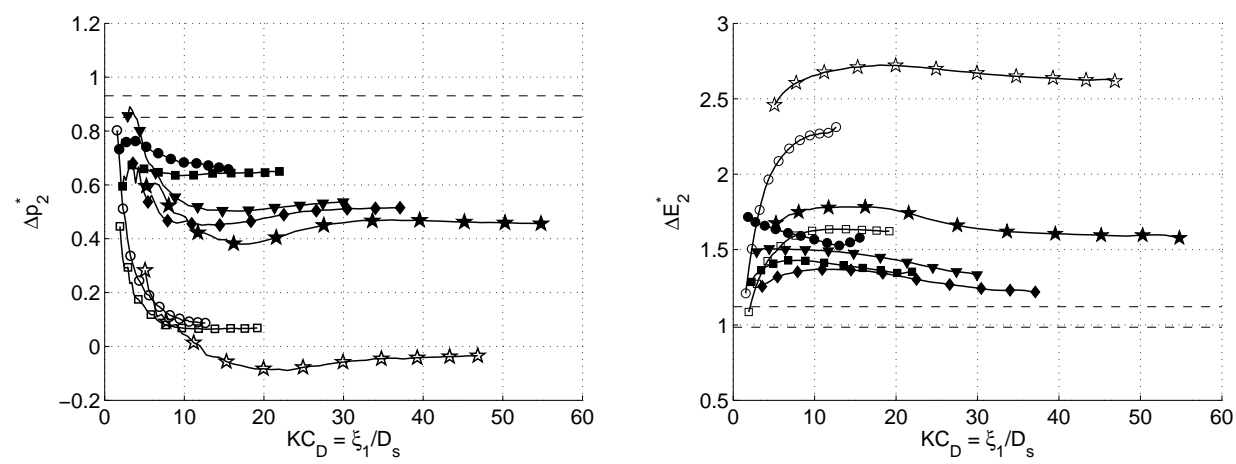

Figure 2: Dimensionless pressure drop (left) and dimensionless acoustic power dissipation (right) for all jet pump samples. Solid markers correspond to $7^{\circ}$ taper angle, open markers to $15^{\circ}$ taper angle. Different marker types are used to indicate the number of holes: $1(\bullet), 2(\mathbf{\nabla}), 4(\mathbf{\nabla}), 8(\diamond)$ and $16(\star)$. For clarity, markers are shown only in one fifth of the measured points. Dashed lines represent bounds of values calculated using the quasi-steady approximation.

\section{Conclusions and outlook}

Switching from single to multiple hole jet pump geometries certainly has an effect on the performance in terms of the time-averaged pressure drop and acoustic power dissipation. A lower dimensionless time-averaged pressure drop is measured when increasing the number of holes. Possible causes for this phenomenon are the interaction between jets from the different holes and the effect of turbulence on the jet pump performance. The latter will be investigated using hot-wire anemometry and flow visualization.

\section{Acknowledgements}

The authors would like to gratefully thank Jos Zeegers and the Eindhoven University of Technology for the provision of the experimental apparatus. Bosch Thermotechnology and Agentschap NL are thankfully acknowledged for the financial support as part of the EOS-KTO research program under project number KTOT03009.

\section{References}

[1] Aben, P. C. H. High-Amplitude Thermoacoustic Flow Interacting with Solid Boundaries. Phd thesis, Technische Universiteit Eindhoven (2010).

[2] Backhaus, S. and Swift, G. "A thermoacoustic-Stirling heat engine: detailed study". J. Acoust. Soc. Am., 107, (2000), 3148-66.

[3] Biwa, T., Tashiro, Y., Ishigaki, M., Ueda, Y., and Yazaki, T. "Measurements of acoustic streaming in a looped-tube thermoacoustic engine with a jet pump". Journal of Applied Physics, 101, (2007), 064914.

[4] Oosterhuis, J. P., Bühler, S., Wilcox, D., and Van der Meer, T. H. "A numerical investigation on the vortex formation and flow separation of the oscillatory flow in jet pumps". J. Acoust. Soc. Am., 137, (2015), 1722-1731.

[5] Smith, B. L. and Swift, G. W. "Power dissipation and time-averaged pressure in oscillating flow through a sudden area change". J. Acoust. Soc. Am., 113, (2003), 2455-2463. 